\title{
Standard and consistent Detached-Eddy Simulation for tur- bulent engine flow modeling: an application to the TCC-III engine
}

\author{
Vesselin Krassimirov Krastev ${ }^{1, *}$, Giovanni Di Ilio ${ }^{2}$, Clara Iacovano $^{3}$, Alessandro d'Adamo ${ }^{3}$, \\ and Stefano Fontanesi ${ }^{3}$ \\ ${ }^{1}$ Department of Enterprise Engineering "Mario Lucertini", University of Rome "Tor Vergata" Via del \\ Politecnico, 1 - 00133 Rome (Italy) \\ ${ }^{2}$ Department of Engineering, University of Naples "Parthenope" Centro Direzionale, Isola C4 - 80133 \\ Naples (Italy) \\ ${ }^{3}$ DIEF - "Enzo Ferrari" Engineering Department, University of Modena and Reggio Emilia \\ Via Vivarelli, 10 - 41125 Modena (Italy)
}

\begin{abstract}
Multidimensional modeling of Cycle-to-Cycle Variability (CCV) has become a crucial support for the development and optimization of modern direct-injection turbocharged engines. In that sense, the only viable modeling options is represented by scale-resolving approaches such as Large Eddy Simulation (LES) or hybrid URANS/LES methods.

Among other hybrid approaches, Detached-Eddy Simulation (DES) has the longest development story and is therefore commonly regarded as the most reliable choice for engineering-grade simulation. As such, in the last decade DESbased methods have found their way through the engine modeling community, showing a good potential in describing turbulence-related CCV in realistic engine configurations and at reasonable computational costs.

In the present work we investigate the in-cylinder modeling capabilites of a standard two-equation DES formulation, compared to a more recent one which we call DESx. The DESx form differs from standard DES in the turbulent viscosity switch from URANS to LES-like behavior, which for DESx is fully consistent with Yoshizawa's one-equation sub-grid scale model. The two formulations are part of a more general Zonal-DES (ZDES) methodology, developed and validated by the authors in a series of previous publications. Both variants are applied to the multi-cycle simulation of the TCC-III experimental engine setup, using sub-optimal grid refinement levels in order to stress the model limitations in URANS-like numerical resolution scenarios. Outcomes from this study show that, although both alternatives are able to ouperform URANS even in coarse grid arrangements, DESx emerges as sligthly superior and thus it can be recommended as the default option for in-cylinder flow simulation.
\end{abstract}

\section{Introduction}

After thirty years of development, hybrid URANS/LES turbulence models are considered a powerful and reliable tool for the numerical description of a large variety of turbulent flows.

\footnotetext{
*e-mail: krastev@dii.uniroma2.it
} 
Since the seminal work of Spalart et al. [1], which was focused on wing aerodynamics, hybrid methods have been continuously refined and extended for the simulation of engineering systems of increasing complexity. Nonetheless, the basic rationale behind merging the Reynolds-Averaged and Large Eddy Simulation formalisms still remains the same: combining the best features of both approaches, depending on the available numerical resolution and discretization quality.

Aside from wing aerodynamics, current typical examples of engineering-grade hybrid URANS/LES applications include full-scale turbomachinery, ship hydrodynamics, aeroacoustics, large-scale urban flows and jet propulsion [2-6]. Less common, but with a rapidly evolving track record in the past ten years, is the implementation of hybrid URANS/LES models for the scale-resolving simulation of flow through internal combustion engines [7]. The main reason for considering hybrid approaches for engine modeling is the increasingly compelling need for high-quality time and space resolved numerical data sets, but at a reduced computational cost compared to standard LES, as outlined in [8-11]. Most of the studies currently reported in the scientific literature are based on the Detached-Eddy Simulation (DES) concept and its derivatives [12-27], while a few of them relies on the Scale-Adaptive Simulation (SAS) approach [15, 16, 28, 29].

In the present paper we evaluate the most recent improvements to a zonal DES-based methodology, which has been developed in a series of previous publications for the simulation of full-scale internal combustion engine configurations. The purpose here is to stress the subgrid scale model consistency whenever the LES mode is enabled by the hybrid method, with a special focus on coarse (URANS-like) grid resolution scenarios. The analysis is made on the TCC-III reference experimental engine [30], which represents an excellent sample of realistic engine geometry and operating conditions. The remainder of the paper includes two more sections, one devoted to a brief description of the method and equations involved and the last one showing the simulation setup and results.

\section{Turbulence modeling}

\subsection{Standard and consistent RNG k- $\varepsilon$ DES formulations}

A standard DES methodology belongs to the so-called seamless (or universal) hybrid modeling category [4]. Seamless approaches are generally independent from the specific underlying URANS turbulence model and allow the automatic switching between URANS and LES (and vice-versa), depending on the local flow conditions. In our case, the starting point is represented by compressible RNG $k-\varepsilon$ URANS transport equations:

$$
\begin{gathered}
\rho \frac{\partial k}{\partial t}+\nabla \cdot(\rho \mathbf{U} k)=\rho(\mathcal{P}-\varepsilon)+\nabla \cdot\left[\left(\frac{\mu_{t}}{\sigma_{k}}+\mu\right) \nabla k\right] \\
\rho \frac{\partial \varepsilon}{\partial t}+\nabla \cdot(\rho \mathbf{U} \varepsilon)=\rho \frac{\varepsilon}{k}\left(C_{\varepsilon 1} \mathcal{P}-C_{\varepsilon 2} \varepsilon\right)-\rho R+C_{\varepsilon 3} \rho \varepsilon(\nabla \cdot \mathbf{U})+\nabla \cdot\left[\left(\frac{\mu_{t}}{\sigma_{\varepsilon}}+\mu\right) \nabla \varepsilon\right] \\
\mu_{t}=\rho C_{\mu} \frac{k^{2}}{\varepsilon}
\end{gathered}
$$

with

$$
R=C_{\mu} \eta^{3} \frac{\left(1-\eta / \eta_{0}\right)}{1+\zeta \eta^{3}} \frac{\varepsilon^{2}}{k} ; \quad \eta=|\mathbf{E}| \frac{k}{\varepsilon}
$$


The standard DES modification of a two-equation URANS model requires only a minor change in the turbulent kinetic energy destruction term $\mathcal{S}_{k}[31]$, that is:

$$
\begin{gathered}
\mathcal{S}_{k, R A N S}=\rho \varepsilon=\rho \frac{k^{3 / 2}}{l_{R A N S}} ; \quad l_{R A N S}=\frac{k^{3 / 2}}{\varepsilon} \\
\mathcal{S}_{k, D E S}=F_{D E S} \mathcal{D}_{R A N S} ; \quad F_{D E S}=\max \left(\frac{l_{\text {RANS }}}{C_{D E S} \Delta}, 1\right)
\end{gathered}
$$

where $\Delta$ is a grid-related filter length scale and $C_{D E S} \approx O(1)$. The $\Delta$ parameter is calculated as the cubic root of the computational cell volume, while $C_{D E S}$ is set to 0.61 [27]. As stated above, the expressions (5) and (6) are general and can be applied to any URANS form, by just adapting $l_{\text {RANS }}$ accordingly.

When in LES mode $\left(F_{D E S}>1\right)$, Equation (6) is known to produce an inertial sub-range $\mu_{t}$ scaling that is consistent with a Smagorinsky-like LES model [31]. Nonetheless, even in that case the turbulence model retains its two-equation nature, which has no rigorous theoretical foundation except for the special case in which the production/destruction mechanisms of both $k$ and $\varepsilon$ (assumed as sub-grid quantities) are in simultaneous equilibrium [32].

To amend this deficiency, we have developed an alternative seamless DES formulation called DESx [25] and based on the X-LES approach from Kok et al. [33]. The $F_{D E S}$ function remains unchanged, but in DESx it modifies also the turbulent viscosity definition, like in the following:

$$
\mu_{t, D E S x}=\rho C_{\mu} \frac{k^{2}}{\varepsilon} \cdot F_{D E S}^{-1}
$$

In this way, when the LES mode is activated $\mu_{t}$ depends only on the length scale $\Delta$ and $k^{1 / 2}$, which is consistent with the physics-based one-equation model of Yoshizawa and Horiuti [34].

\subsection{Zonal-DES implementation}

The zonal implementation of $F_{D E S}$ requires the following rearrangement [18]:

$$
\begin{aligned}
& F_{D E S}^{*}=C_{z 1} F_{D E S}+\left(1-C_{z 1}\right) F_{Z D E S} \\
& F_{Z D E S}=C_{z 2}+\left(1-C_{z 2}\right)\left(\frac{l_{R A N S}}{C_{D E S} \Delta}\right)
\end{aligned}
$$

where $F_{D E S}^{*}$ should replace $F_{D E S}$ in (6) and (7). Depending on the available combinations of the boolean parameters $C_{z 1}$ and $C_{z 2}$, the user can define a priori the URANS, LES or seamless DES/DESx mode of operation in different zones of the computational domain (see Table 1).

Table 1: Modes of operation of $F_{D E S}^{*}$.

\begin{tabular}{ccc}
\hline$C_{z 1}$ & $C_{z 2}$ & Simulation type \\
\hline 0 & 1 & URANS \\
0 & 0 & LES \\
1 & 1,0 & DES or DESx \\
\hline
\end{tabular}




\section{TCC-III engine simulations}

\subsection{Case setup}

The TCC-III is a widely documented optical access engine benchmark [30], with a vertical two-valve design, a pancake-shaped combustion chamber and large intake and exhaust plenum volumes. The bore $\times$ stroke values are equal to $92 \times 86 \mathrm{~mm}$, with a (nominal) compression ratio of 10:1. This engine has already been the object of extensive numerical studies by the authors $[26,27,35,36]$, in which the zonal hybrid methodology shown above has been implemented into the STAR-CD software [37] and then assessed and validated on a relatively fine computational grid $\left(2 \times 10^{6}\right.$ elements at the BDC).

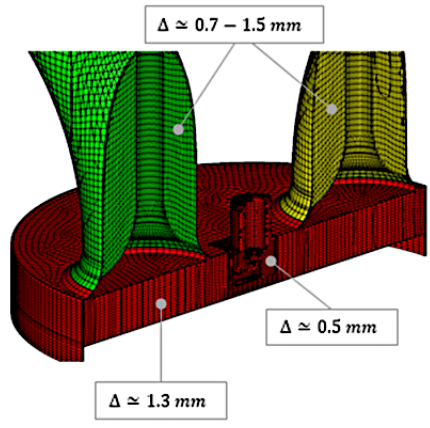

(a)

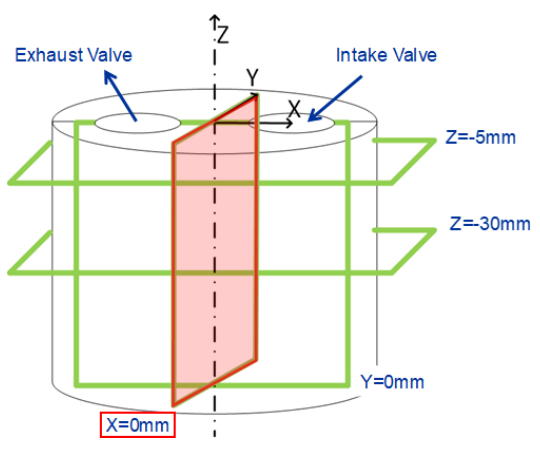

(b)

Figure 1: a) grid density distribution in the cylinder and intake/exhaust ports of the TCC-III computational model laser; $\mathrm{b}$ ) sheet locations taken from [30] (the $\mathrm{x}=0$ plane is highlighted in red).

Nonetheless, a head-to-head comparison between the DES and DESx modes of operation was still missing and is, therefore, proposed here. To perform this investigation, a coarser computational grid has been generated, with a maximum of $\approx 1 \times 10^{6}$ elements at the BDC. The full engine geometry has been included in the model, with the in-cylinder zone (colored in red in Figure 1a) treated as DES or DESx and all the remaining volumes treated as URANS. Further details on the numerical method and setup can be found in [26, 27] and are omitted here for the sake of brevity.

\subsection{Results}

The reference experimental measurement campaign is based on four laser-sheet PIV locations, which are depicted in Figure 1b. In the present study, we focus the attention on the $\mathrm{x}=$ 0 plane, which represents a cross-cut of the large intake vortex structures and is, therefore, the most suitable for a careful analysis of the three-dimensional flow evolution. The computed numerical data sets include 50 consecutive engine cycles, with the flow field evaluated at three selected crank angle values, that is: 475 degrees, equivalent to the intake valve maximum lift; 540 and 630 degrees, equivalent to BDC and mid-compression stroke, respectively. Statistics from 50 cycles is extracted also from the experimental data base, while a converged full URANS solution on the same grid is also included for evaluation. The comparison has been made in terms of Ensamble-Averaged and RMS in-plane velocity fields and complemented by the derivation of synthetic quality indices for each reference CA. The latter are based on the so-called Relevance Index $(R I)$ and Magnitude Index (MI) definitions [29], namely: 


$$
R I_{k}=\frac{\left(\mathbf{U}_{k} \cdot \mathbf{V}_{k}\right)}{\left\|\mathbf{U}_{k}\right\| \cdot\left\|\mathbf{V}_{k}\right\|} ; \quad M I_{k}=1-\frac{\left\|\mathbf{U}_{k}-\mathbf{V}_{k}\right\|}{\left\|\mathbf{U}_{k}\right\|+\left\|\mathbf{V}_{k}\right\|}
$$

with $k$ being the generic data sampling point and $\mathbf{U}_{k}, \mathbf{V}_{k}$ being the measured and computed velocity vector evaluated at the $k-t h$ point. The $R I_{k}$ parameter is a local estimator of the orientation consistency between the $\mathbf{U}_{k}$ and $\mathbf{V}_{k}$ vectors and its value may vary between 1 (same orientation) and -1 (opposite orientation). The $M I_{k}$ parameter is a combined orientation/magnitude local estimator and may vary between 1 (full equivalence of magnitude and orientation) and 0 (opposite orientation). The local $R I$ and $M I$ values can be then planeaveraged, in order to obtain a single synthetic indicator that is representative of a specific $C A$ and Field Of View (FOV), that is:

$$
\overline{R I}=\frac{1}{N} \sum_{k=1}^{N} R I_{k} ; \quad \overline{M I}=\frac{1}{N} \sum_{k=1}^{N} M I_{k}
$$

with $N$ being the total number of sampling points over the FOV. Expressions (11) can be enhanced with the addition of Kinetic Energy (KE)-based weights in the averaging operator [26]:

$$
\overline{R I}_{w a}=\frac{\sum_{k=1}^{N} R I_{k} \cdot K E_{k}}{\sum_{k=1}^{N} K E_{k}} ; \quad \overline{M I}_{w a}=\frac{\sum_{k=1}^{N} M I_{k} \cdot K E_{k}}{\sum_{k=1}^{N} K E_{k}}
$$

with:

$$
K E_{k}=\frac{1}{2} \cdot\left(\left|\mathbf{U}_{k}\right|^{2}+\left|\mathbf{V}_{k}\right|^{2}\right)
$$

Expressions (12) are believed to return a more statistically relevant global assesment of $R I$ and $M I$, by suppressing the relevance of the low-speed/low-energy data which are likely to possess the highest relative uncertainity levels.

Figures 2-4 show in-cylinder Ensemble-Averaged results from URANS, DES and DESx, compared to the experiments. The benefits of using a scale-resolving (DES or DESx) approach instead of a Reynolds-Averaged one are apparent, even with an average grid resolution that is well below the standard of a high-quality LES. Differences between DES and DESx are less obvious, but the flow topology returned by DESx seems to be slightly more consistent with the experiments, especially during the compression stroke (at $540 \mathrm{CA}$ and at $630 \mathrm{CA}$ ). The RMS fields (Figures 5-7 basically confirm the slight qualitative advantage of DESx at 630 CA. Observations from the averaged $R I$ and $M I$ levels (Figures 8-9) allow a more systematic differentiation between the two hybrid formulations. Focusing on the KE-weighted average, values at $475 \mathrm{CA}$ and $540 \mathrm{CA}$ are all very close one to each other. Conversely, at 630 CA the DESx model produces a $+2.2 \%$ and a close to $+5 \%$ increase in the $\overline{R I}_{w a}$ and $\overline{M I}_{w a}$ levels, respectively. Remarkably, for both models $\overline{R I}_{w a}$ are always above 0.9 , which means that at least the Ensemble-Averaged qualitative flow topology is well captured at different CAs.

\section{Conclusions}

In this study two DES formulations are compared and applied to the compressible RNG $k-\varepsilon$ URANS turbulence model: a standard DES and an improved version of the same model, fully consistent with a one-equation sub-grid scale model (called DESx). The application to a full engine case (the TCC-III) under motored conditions and the comparison of simulated fields against 2D PIV confirms that: 
- the usage of robust scale-resolving turbulence models represents a key improvement for multi-cycle engine simulation, compared to standard Reynolds-Averaged models;

- the above is true even for coarse-grid configurations, thus prioritizing the attention of engine CFD researchers on the adoption/development of advanced hybrid models in order to effectively optimize the available computational resources and turnaround times;

- compared to standard DES, the DESx formulation have shown more accurate results at no additional complexity or computational cost, thus rendering it the recommended standard for in-cylinder engine simulations of highly turbulent flows.

Concerning the total computational overhead of DES/DESx compared to standard URANS, the two modeling approaches are not directly comparable due to the low cycle count required by URANS for statistical convergence. If a single cycle computation is considered, the overhead can be estimated at $20-30 \%$, due to the larger gradients and flow instabilities that has to be accounted for by the algebraic solution algorithms.

\section{Acknowledgments}

Siemens PLM Software Inc. is kindly acknowledged for providing the STAR-CD software licensing, through the HPC resources of the University of Modena and Reggio Emilia.

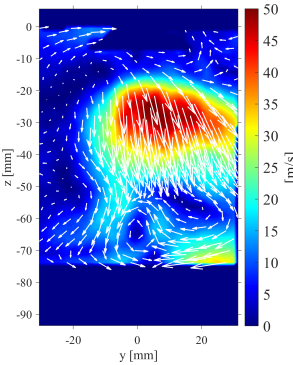

(a)

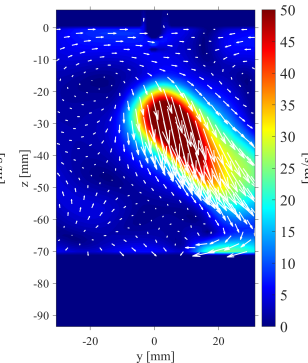

(b)

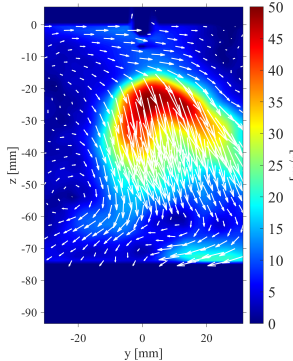

(c)

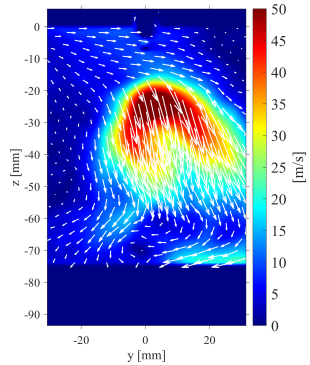

(d)

Figure 2: Ensemble-averaged velocity fields at $475 \mathrm{CA}, \mathrm{x}=0$ : a) PIV [30]; b) URANS; c) DES; d) DESx.

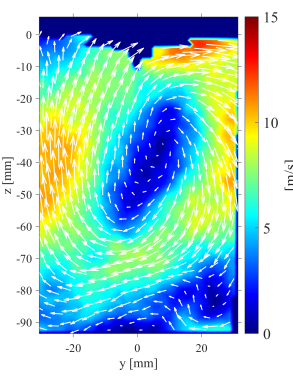

(a)

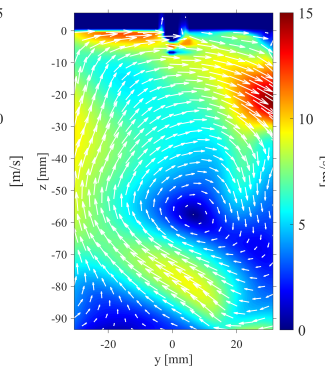

(b)

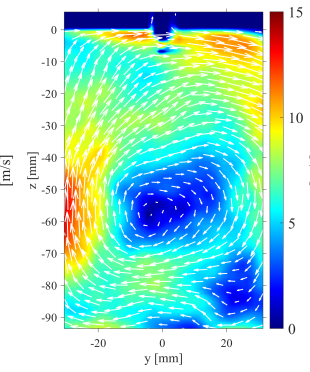

(c)

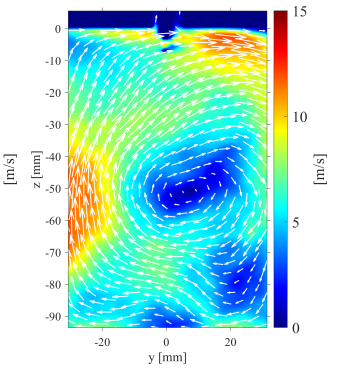

(d)

Figure 3: Ensemble-averaged velocity fields at $540 \mathrm{CA}, \mathrm{x}=0$ : a) PIV [30]; b) URANS; c) DES; d) DESx. 


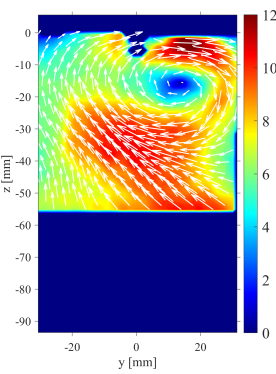

(a)

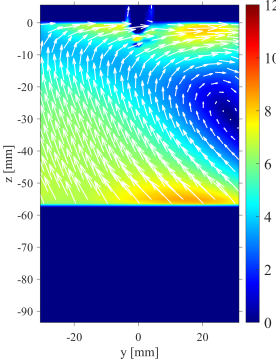

(b)

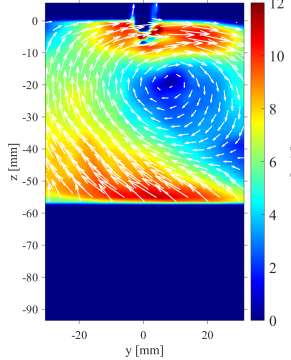

(c)

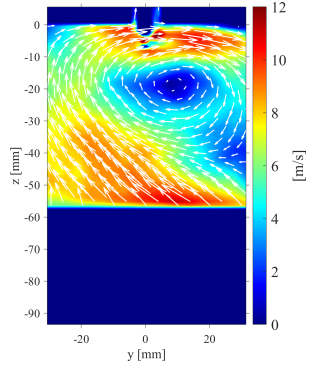

(d)

Figure 4: Ensemble-averaged velocity fields at $630 \mathrm{CA}, \mathrm{x}=0$ : a) PIV [30]; b) URANS; c) DES; d) DESx.

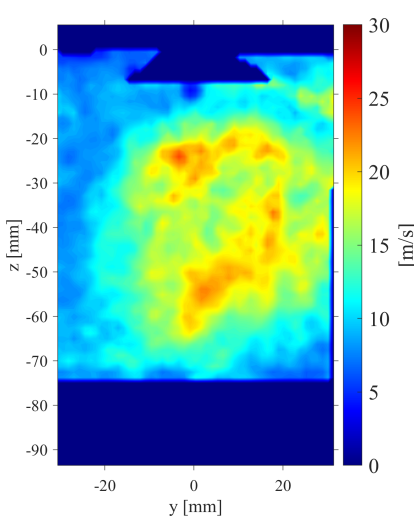

(a)

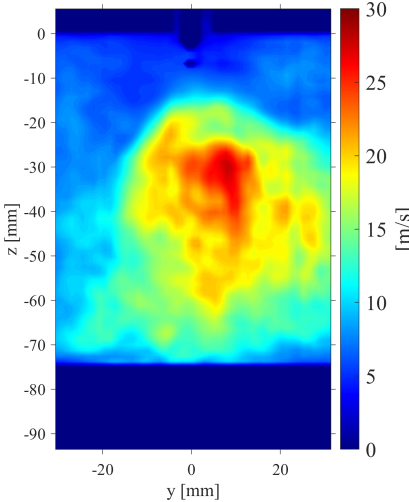

(b)

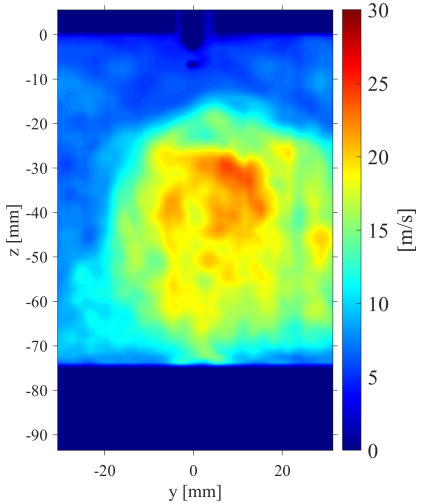

(c)

Figure 5: RMS velocity fields at 475 CA, $x=0$ : a) PIV [30]; b) DES; c) DESx.

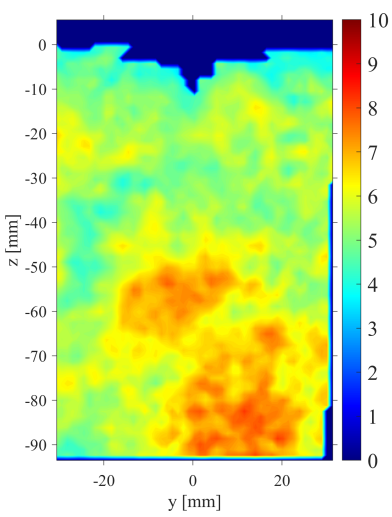

(a)

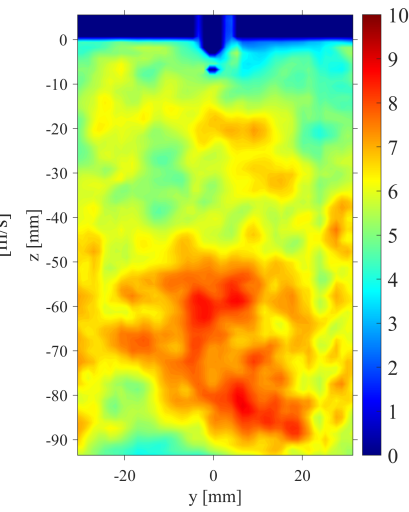

(b)

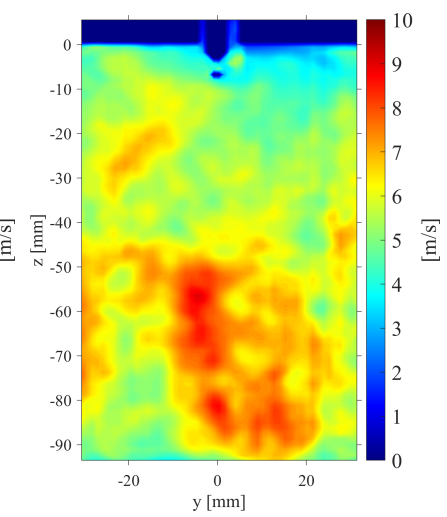

(c)

Figure 6: RMS velocity fields at $540 \mathrm{CA}, \mathrm{x}=0$ : a) PIV [30]; b) DES; c) DESx. 


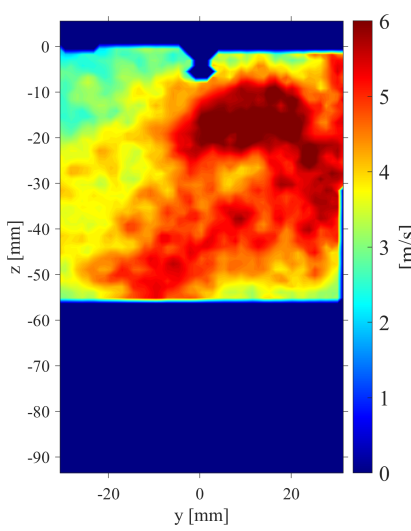

(a)

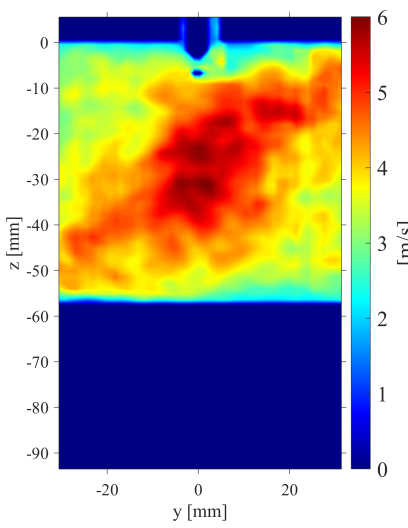

(b)

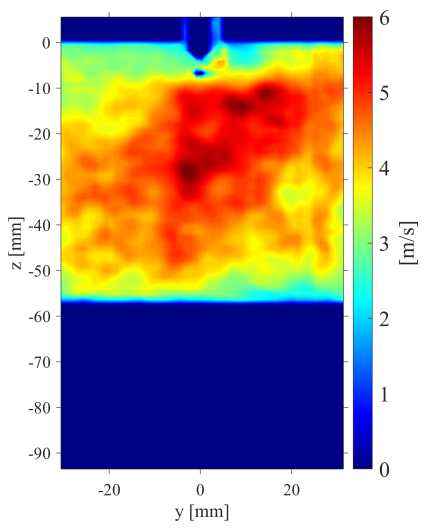

(c)

Figure 7: RMS velocity fields at 475 CA, $x=0$ : a) PIV [30]; b) DES; c) DESx.

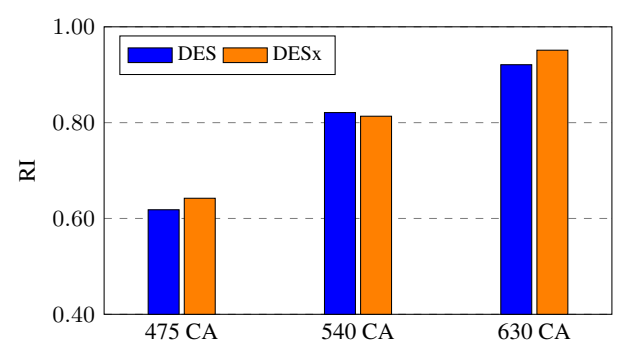

(a)

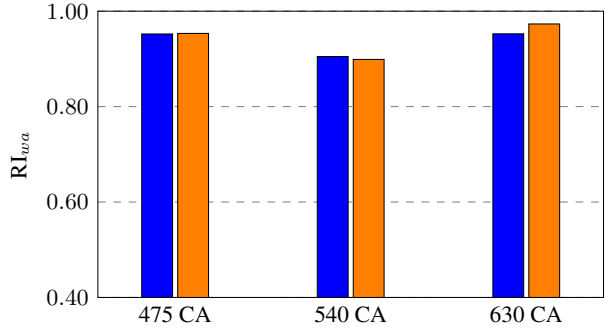

(b)

Figure 8: Averaged in-plane values of the Relevance Index a) standard average; b) KE-weighted average.

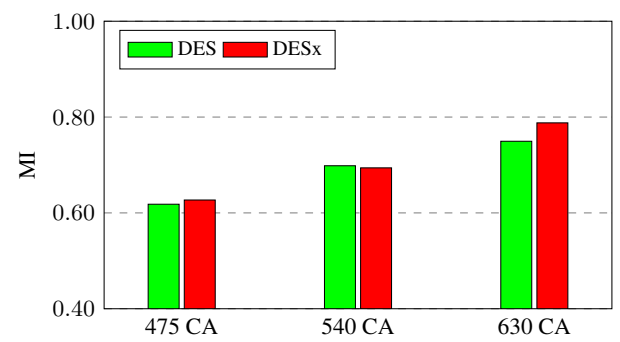

(a)

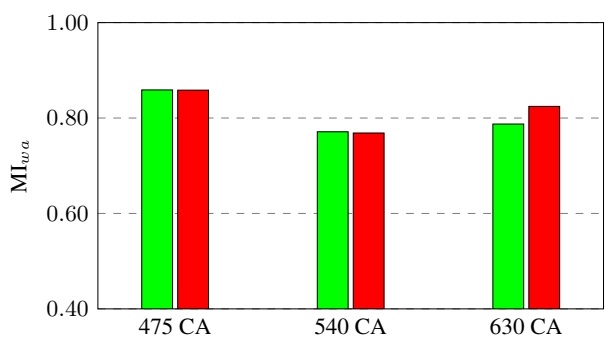

(b)

Figure 9: Averaged in-plane values of the Magnitude Index a) standard average; b) KE-weighted average.

\section{References}

[1] P.R. Spalart, W.H. Wou, M. Strelets, S.R. Allmaras, in Advances in DNS/LES, edited by C. Liu, Z. Liu (Greyden Press, Columbus, Ohio, USA, 1997), pp. 137-147

[2] P.G. tucker, Progress in Aerospace Sciences 47, 546 (2011) 
[3] P.G. tucker, S. Eastwood, C. Klostermeier, H. Xia, P. Ray, J. Tyacke, W. Dawes, Journal of Turbomachinery 134, 021024 (2012)

[4] P. Sagaut, S. Deck, M. Terracol, Multiscale and multiresolution approaches in turbulence - LES, DES and Hybrid RANS/LES Methods: Applications and Guidelines (Imperial College Press, 2013), ISBN 978-1-84816-986-9

[5] P.G. tucker, J.R. DeBonis, Phil. Trans. R. Soc. A 372, 20130331 (2014)

[6] Y. Hoarau, S.H. Peng, D. Schwamborn, A. Revell, C. Mockett, eds., Progress in Hybrid RANS-LES Modelling - Papers Contributed to the 7th Symposium on Hybrid RANS-LES Methods, 17-19 September, 2018, Berlin, Germany, Notes on Numerical Fluid Mechanics and Multidisciplinary Design (Springer International Publishing, 2018), ISBN 978-3-030-27606-5

[7] V.K. Krastev, G. Di Ilio, G. Falcucci, G. Bella, Energy Procedia 148, 1098 (2018)

[8] Y. Shekhawat, D. Haworth, A. d'Adamo, F. Berni, S. Fontanesi, P. Schiffmann, D. Reuss, V. Sick, Oil \& Gas Science and Technology - Rev. IFP 72 (2018)

[9] A. D’Adamo, S. Breda, G. Cantore, Energy Procedia 82, 45 (2015)

[10] A. D'Adamo, S. Breda, F. Berni, S. Fontanesi, SAE Int. J. Engines 12, 22 (2019)

[11] A. D’Adamo, S. Breda, F. Berni, S. Fontanesi, Applied Energy 249, 126 (2019)

[12] C. Hasse, V. Sohm, B. Durst, International Journal of Heat and Fluid Flow 30, 32 (2009)

[13] C. Hasse, V. Sohm, B. Durst, Computers \& Fluids 39, 25 (2010)

[14] C. Hasse, International Journal of Engine Research 17, 44 (2016)

[15] S. Buhl, F. Dietzsch, C. Buhl, C. Hasse, Computers \& Fluids 156, 66 (2017)

[16] S. Buhl, D. Hain, F. Hartmann, C. Hasse, International Journal of Engine Research 19, $282(2018)$

[17] V.K. Krastev, G. Bella, G. Campitelli, SAE Technical Paper 2015-24-2414. (2015)

[18] V.K. Krastev, G. Bella, SAE Int. J. Engines 9, 1425 (2016)

[19] V.K. Krastev, L. Silvestri, G. Falcucci, G. Bella, SAE Technical Paper 2017-24-0030. (2017)

[20] V.K. Krastev, L. Silvestri, G. Falcucci, Energies 10, 2116 (2017)

[21] V.K. Krastev, L. Silvestri, G. Bella, SAE Int. J. Engines 11, 669 (2018)

[22] G. Di Ilio, V.K. Krastev, F. Piscaglia, G. Bella, SAE Technical Paper 2019-01-0270 (2019)

[23] G. Di Ilio, V.K. Krastev, G. Falcucci, Energies 12, 2699 (2019)

[24] V.K. Krastev, G. Di Ilio, AIP Conference Proceedings 2191 (2019)

[25] G. Di Ilio, V.K. Krastev, G. Bella, SAE Technical Paper 2019-24-0127. (2019)

[26] V.K. Krastev, A. d'Adamo, F. Rulli, S. Fontanesi, SAE Technical Paper 2019-24-0097. (2019)

[27] V.K. Krastev, A. d'Adamo, F. Berni, S. Fontanesi, International Journal of Engine Research 21, 632 (2020)

[28] S. Buhl, F. Hartmann, C. Hasse, Oil \& Gas Science and Technology - Rev. IFP 71 (2016)

[29] S. Buhl, F. Gleiss, M. Köhler, F. Hartmann, D. Messig, C. Brücker, C. Hasse, Flow Turbulence Combust 98, 579 (2017)

[30] P. Schiffmann, S. Gupta, D. Reuss, V. Sick, X. Yang, T. Kuo, Oil \& Gas Science and Technology - Rev. IFP 71 (2016)

[31] A. Travin, M.L. Shur, M. Strelets, P.R. Spalart, in Advances in LES of Complex Flows, edited by R. Friedrich, W. Rodi (Kluwer Academic Publishers, Netherlands, 2002), pp. 239-254

[32] J. Yan, C. Mockett, F. Thiele, Flow Turbulence Combust 74, 85 (2005) 
[33] J. Kok, H. Dol, H. Oskam, H. Van Der Ven, AIAA Paper 2004-264. (2004)

[34] A. Yoshizawa, K. Horiuti, J. Phys. Soc. Jpn. 54, 2834 (1985)

[35] I. Ko, A. D’Adamo, S. Fontanesi, K. Min, SAE Technical Paper 2017-01-0549 (2017)

[36] I. Ko, K. Min, F. Rulli, A. D’Adamo, F. Berni, S. Fontanesi, SAE Technical Paper 2017-24-0040 (2017)

[37] STAR-CD CFD/CCM User Guide Version 4.28 (Siemens Product Lifecycle Management Inc., 2017) 